\title{
Barriers and Facilitators in Adopting Healthy Lifestyles in West Ethiopia: A Qualitative Research Study
}

\author{
Alemu Adeba 8 (D) $\triangle$, Dessalegn Tamiru 8 (D) and Tefera Belachew ${ }^{3}$ (D) \\ ${ }^{123}$ Department of Nutrition and Dietetics, Faculty of public Health, Jimma University, Ethiopia \\ $\triangle$ Corresponding Author: Alemu Adeba, E-mail: alemuadeba2017@gmail.com
}

\section{ARTICLE INFORMATION}

Received: April 08, 2021

Accepted: June 01, 2021

Volume: 2

Issue: 1

DOI: $10.32996 /$ jmhs.2021.2.1.3

\section{KEYWORDS}

Healthy lifestyles, Adoption, Social capital, Qualitative analysis

\section{ABSTRACT}

Risks of NCDs are prevented by adopting healthy lifestyles, but it remains a matter of concern and the knowledge regarding the effect of social factors on health almost not researched in West Ethiopia. The objective of the study is to explore barriers and facilitators in adopting healthy lifestyles among urban residents of West Ethiopia. A qualitative study design was used with three diversified focus group discussion (FGD) guidance to obtain adoption and perception of 27 participants' on healthy lifestyles. The collected audio-record and session note data's; were transcribed, verbatim and qualitatively analyzed through a thematic approach. The following themes were identified based on social capital perception frames (bonding, bridging, and linking). Barriers theme: negative influence from family, peer pressure and perceive towards unhealthy lifestyles \&obesity, and accepted socialization by the community (bonding); inadequate awareness on NCD screening and management and insufficient support from health care workers (bridging); and hospital \& community healthcare professionals related barriers, inequity in the allocation of public resources (linking). Facilitators theme: motivation to participate in physical activity (bonding); cooperative relationships with health extension workers (bridging); and fear of morbidity and mortality; access of media to an aware, healthy lifestyle (linking); were identified themes to healthy lifestyles adoption. This study revealed that urban residents of west Ethiopia adopted unhealthy lifestyles. Bonding, bridging and linking social capital would be work as barriers and facilitators to adopting healthy lifestyles. Therefore, integrating quoted findings into the chronic care system need attention.

\section{Introduction}

Degenerative diseases are the major public health concerns worldwide, including in Ethiopia. Two-thirds of NCD mortalities occur in low income developing countries that lack health literacy and resources (Abegunde et al. 2007). NCDs, particularly obesity, cardiovascular, diabetes, cancer, hypertension, stroke, respiratory diseases and their risks are the main causes of morbidity and mortality globally (Mensah, 2016; Fatt, 2016; Cowan, et al.2011; WHO, 2015; Daniels, et al.2005). Chronic diseases can be delayed, prevented, or managed through healthy lifestyles (Siddique et al., 2009; WHO, 2010; Dietz et al., 2016).

Lifestyle adoption increases life expectancy by 0.5 years while metformin by 0.2 years. In addition, associated morbidities such as blindness, end-stage renal disease, amputation, stroke, and coronary heart disease were decreased by greater percentages with lifestyle rather than metformin intervention, so lifestyle intervention provided greater health benefits at a lower cost than the metformin intervention (Zhang, et al.2005).

Lifestyle diseases share risk factors similar to prolonged exposure to four main modifiable lifestyle behaviours: smoking, unhealthy diet, alcoholic's abuse, and physical inactivity. However, the combination of the main healthy lifestyle factors: maintaining a healthy weight, exercising regularly, following a healthy diet, and not smoking seems to be associated with as much as an $80 \%$ reduction in the risk of developing the most common and deadly chronic diseases (Engelen, et al.2017).

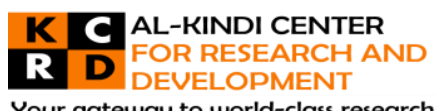

Your gateway to world-class research

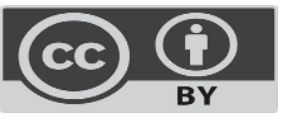

Published by Al-Kindi Center for Research and Development, United Kingdom. Copyright (c) the author(s). This open access article is distributed under a Creative Commons Attribution (CC-BY) 4.0 license 
Studies verified that a healthy diet plus 150 minutes of moderate-intensity, or 75 minutes of vigorous-intensity physical exercise for three to five days per week, incorporating aerobic and strengthening exercises, improves health (Sallis, R.2017; Taylor, D. 2014; WHO; 2004). However, the study conducted on adherence levels to dietary recommendations for chronic care was poor among Ethiopian patients. For instance, in Addis Ababa 51.4\% of poor diet adherence were registered (Worku A, et al.2015), while high percent $(74.3 \%)$ of poor adherence to dietary recommendations among patients with T2DM in Northern Ethiopia (Ayele et al.2018).

Other bottlenecks to adopting healthy lifestyles were lack of physical activity protocol, no skill and standard receipts. Because of these, our people use unstandardized doses of salt, sugar and other substances. For example, standard doses of alcohol for male are two standard drinkings, while one for female. Thus, two standard drinks are equivalent to $25 \mathrm{ml}$ spirits (like whisky, vodka, brandy) or one standard glass $(125 \mathrm{ml})$ wine or $340 \mathrm{ml}$ beer. The main recommendations for a healthy diet are sufficient daily consumption of fruits and vegetables $(\geq 400 \mathrm{~g})$ and restricted consumption of free sugars $(<10 \%$ or, ideally, $<5 \%$ of total daily energy intake), fats ( $<30 \%$ of total daily energy intake from total fats, $<10 \%$ total daily energy intake from saturated fats) and salt $(<6 \mathrm{~g}$ daily or one teaspoon) (WHO; 2016).

In Ethiopia, the epidemiological, behavioural risk factors (tobacco use, alcohol consumption, and khat chewing) showed that $7 \%$ of men use tobacco products; $45 \%$ of women and $53 \%$ of men reported drinking alcohol in their lifetime; $11 \%$ of women and $28 \%$ of men reported that they have ever chewed khat $(E D H S, 2011)$. Another study in Addis Ababa reveals the prevalence of Mets was $14.0 \%$ in men and $24.0 \%$ in women (Girum, 2014). In addition, elevated blood pressure is more common in females (44.5\%) than in males (34.9\%) (Abda et al., 2016).

The impact of unhealthy lifestyles undermines the quality of life, life expectancy, and productivity and contributes to death and economic losses. Escalating rates of obesity in the U.S. have resulted in higher direct and indirect economic costs. Direct medical costs include prevention, diagnosis, and treatment related to obesity. Indirect costs are further subdivided into morbidity and mortality costs. Morbidity costs represent lost income from reduced productivity, restricted physical activity, absenteeism, and bed days. Mortality costs encompass lost future income due to premature death (Spark, Arlene, 2007; Hirakawa, et al. 2016).

In our country, engaging the community into healthy lifestyle adoption was low and also health and nutrition information desk for awaking community on it is not sated yet. The population debunk of the myths and misconceptions of the diseases are other barrier wings. For example, NCDs are the disease of:

"Rich and affluent; ....,Gibto Areki and moringa cure hypertension...; other bottlenecks".

Several qualitative studies have been conducted in Ethiopia to explore people's lifestyles behaviour with regard to communicable diseases. However, no qualitative studies have ever explored barriers and opportunities to adopt healthy lifestyles and prevent NCD through social capital perspectives, while such studies have been conducted in other countries (Hirakawa, et al. 2016).

The underlying causes of unhealthy lifestyles that induced high NCD risk factors among residents were investigated. In-depth exploration of barriers and facilitators to adopting healthy lifestyles to improve modifiable risk factors of chronic illness needs integrated community, nutritionist/dieticians, physicians, sports expert, teachers, general practitioners, health officer, and nurse's team. For interpretation, we used social capital framework with bonding, bridging, and liking categories to elucidate barriers and facilitators to adopting healthy lifestyles among urban residents of western Ethiopia.

\section{Methods}

\subsection{Study design and area}

This study was designed to explore barriers and facilitators in adopting healthy lifestyles among urban residents in urban residents in west Ethiopia: qualitative analysis through social capital perspectives with the provision of class survey, FGDs and sessions notes. Because of the hub centre for western towns (Assosa, Ambo, Bure-Bahirdar, Metu and Jimma) this study was conducted in Nekemte city, which is located at a distance of $328 \mathrm{~km}$ from Addis Ababa.

\subsection{Participants}

With non-probable sampling, participants were purposely recruited through health extension workers, who knew the residents' lifestyles and social backgrounds well. And the participants were purposively selected from health professionals, known chronic diseases patients, undiagnosed communities, teachers, business bankers, community leader and office workers. According to Degu and Tegbar (2006), a minimum of 27sample size calculated from three groups with 6-12 members each needed among adults 41 to 64years. Homogenous group composition was divided into three age-stratified groups with the concept of life expectancy and biological change with regard to menopause age: [41-48, 49-56 and 57-64] years old with comprised of 10, 9 and 8 participants respectively keeping the data saturation. In addition, three individuals experienced in qualitative research methods involved in leading, collecting and analyzing the focus group discussions to do sound research.

\subsection{Analysis}


To manipulate the analysis, cascading the number of participants by code who mentioned a particular theme was performed as the best indicator of prevalent themes (Thairu, et al, 2007). Audio-recorded files were transcribed. Each session notes and focus group data were entered into Microsoft Word for open coding analysis by three coders. To illustrate the analysis, the participants' audio and direct quotes are transcribed into English verbatim, serving as a description of the topic explored. The qualitative data were analysed through social capital perspectives with bonding, bridging and linking frameworks.

\subsection{Focus Group Discussions}

Three focus group discussions (FGDs) were conducted in February 2019 at chelelik clinic compound. A FGD guide was designed to cover the common scale of healthy lifestyle adoption, containing five main domains (Table 1).

Table 1. FGDs guideline to assess the status of healthy lifestyles adoption

\begin{tabular}{|c|c|}
\hline Domains & Probe/Questions \\
\hline (1) Dietary diversity & Food groups (what, how often, role of DD, when, \& where)? \\
\hline (2) Physical activities & PA (Why, how often, effect on health, \& where)? \\
\hline $\begin{array}{l}\text { (3) Substance use (Smoking, alcohol, } \\
\text { cannabises, Chat etc.) }\end{array}$ & $\begin{array}{l}\text { Doses and responses?, Effect on social health? What are the barriers and } \\
\text { facilitators to use or stop? }\end{array}$ \\
\hline (4) Health seeking behaviours & Why seek?, where get and by whom you cared? Any barrier? \\
\hline $\begin{array}{l}\text { (5)Awareness \& perceptions to } \\
\text { prevention \& care of NCDs }\end{array}$ & $\begin{array}{l}\text { Do you know NCD?, mention barriers to adopt healthy lifestyles, your } \\
\text { perception to early detection and care of NCD? }\end{array}$ \\
\hline
\end{tabular}

This guideline provided the arbitrator with the framework for raising topics, and participants were expected to express their perception and practices of adopting healthy lifestyles or not and discuss what barriers and opportunities to adopt healthy lifestyles are. All the FGDs were conducted in Afan Oromo and moderated by Amharic in the selected discussion room. Evidence was captured in photo, audio-recorded and key issues raised in the discussions were noted, and discussion continued until no new issues were raised, or saturated for 60-90 minutes.

Text data were analyzed by thematic approach, which contains the following steps: 1) Audio and notes translated into English verbatim including direct quotes; 2) Familiarization (the principal investigator was read thoroughly and repeatedly the original Afan Oromo transcript to get the concept); 3) Identifying and defining themes guided by study objective; 4) Generating initial codes; 5) Narrating and reviewing themes; 6) Reporting the findings.

\subsection{Familiarization}

The first author repeatedly read the original Afan Oromo transcript to obtain the whole sense of the FGDs. Expert of English linguist read the summary of FGDs translated by qualitative data researchers and analyzers.

\subsection{Generating initial codes}

The first author generated the initial codes from the original Afan Oromo transcript. Then those initial codes were translated into English and shared with all the analysts. And both promoters reconciled differences and finalized the coding scheme.

This study, the coding process hold both inductive and deductive approaches. Initial coding was performed by assigning cohesive chunks of text to broadly explore the opportunities and Barriers to adopting healthy lifestyles. During this process, we found that most of the findings of the study were booming with social capital theory. Thus, we applied the social capital framework to investigate the additional codes.

In the present study, social capital is a set of shared values that allows individuals in a group to work together effectively to achieve a common purpose, and it is an appositive product of human interaction (Will Kenton, 2019). Other scholars defined social capital as "social connections and the attendant norm and trust" and classified it as bonding, bridging, and linking (Putnam RD, 1995; Szreter \& Woolcock, 2004). Bonding social capital refers to the strong ties with individuals who are homogeneous in social composition (have similar interest and goal), whereas bridging social capital refers to the weak ties with individuals who are heterogeneous in social composition. In this study, we defined bonding as strong ties with family or close 
friends and bridging as the networks in the community level. Linking social capital was defined as the district, provincial and national level issues, such as the vertical ties with people with the local and central government.

\subsection{Identifying and Defining themes}

Themes were finally created to link the categories and to map the whole thematic system. Table 2, illustrates the process of themes generated from the transcribed text by showing some examples and reviews the trial example of generating themes from the text

\begin{tabular}{|c|c|c|c|}
\hline FGDs & Group of age $41-48$ & Group of age $49-56$ & Group of age $57-64$ \\
\hline Text & $\begin{array}{l}\text { "I have no choice...we are } \\
\text { good friends, especially } \\
\text { those who have been so for } \\
\text { a long time. If they drink } \\
\text { more than two bottles of } \\
\text { alcohol, how can l just drink } \\
\text { half?" }\end{array}$ & $\begin{array}{l}\text { "I know smoking is } \\
\text { harmful,.... fill in a good } \\
\text { mood, when I smoke full } \\
\text { packet." }\end{array}$ & $\begin{array}{l}\text { "I really hope that...of course you can do an } \\
\text { intervention here...I hope that you } \\
\text { professionals could give us acceptable and } \\
\text { feasible } \\
\text { Suggestions to make us healthier. We need } \\
\text { more knowledge to change our lifestyles. We } \\
\text { all still like to put a lot of oil and salt when } \\
\text { cooking. We know we can not drink too } \\
\text { much, but we still like drinking." }\end{array}$ \\
\hline Codes & $\begin{array}{l}\text { I need to drink a lot to keep } \\
\text { a good relationship with } \\
\text { friends. }\end{array}$ & $\begin{array}{l}\text { There is a lack of chronic } \\
\text { diseases health care } \\
\text { workers in the community. }\end{array}$ & $\begin{array}{l}\text { We have some know-how about healthy } \\
\text { lifestyles, but we don't know how to do it. We } \\
\text { did not have procedures/protocols }\end{array}$ \\
\hline $\begin{array}{l}\text { Sub- } \\
\text { category }\end{array}$ & Peer pressure & $\begin{array}{l}\text { Inequity in the allocation of } \\
\text { public resources }\end{array}$ & $\begin{array}{l}\text { Insufficient support from health care } \\
\text { professionals }\end{array}$ \\
\hline category & Bonding social capital & Linking social capital & Bridging social capital \\
\hline Theme & Barriers for the adoption o & healthy lifestyles & \\
\hline Texts & $\begin{array}{l}\text { "Yes! When I was young, I } \\
\text { had to work and look for } \\
\text { physical activity, but since } \\
\text { there is not, it is } \\
\text { impossible. Now, I am } \\
\text { living nearby to engage". }\end{array}$ & $\begin{array}{l}\text { "In the last decade I didn't } \\
\text { know it (healthy lifestyle), } \\
\text { now I understand how } \\
\text { important it is. }\end{array}$ & \\
\hline Codes & $\begin{array}{l}\text { Since my home nearby to } \\
\text { a private sports club, it } \\
\text { helped me engaging in } \\
\text { regular exercise }\end{array}$ & $\begin{array}{l}\text { Nowadays, health } \\
\text { knowledge is disseminated } \\
\text { through many ways, such } \\
\text { as TV and radio. }\end{array}$ & \\
\hline $\begin{array}{l}\text { Sub- } \\
\text { category }\end{array}$ & $\begin{array}{l}\text { Motivation to participate in } \\
\text { regular } \\
\text { exercise }\end{array}$ & $\begin{array}{l}\text { Awareness about healthy } \\
\text { lifestyle given nationwide } \\
\text { through media. }\end{array}$ & \\
\hline Category & Bonding social capital & $\begin{array}{l}\text { Linking } \\
\text { capital }\end{array}$ & \\
\hline Theme & \multicolumn{3}{|c|}{ Opportunity for adopting healthy lifestyles } \\
\hline
\end{tabular}

\subsection{Reporting}

Colourful and persuasive examples from participants' conversation in FGDs were selected and presented in the form of quotes for accurately illustrating our findings.

\subsection{Ethical consideration}

The Institutional Review Board approved this study (IRB), Institute of Health, Jimma University (Approval No. IHRPGY/596/2019; January 1 , 2019). Consent of each participant was obtained prior to the study. Data were analyzed anonymously and quotes were showed with participant codes instead of names. 


\section{Results}

\subsection{Participant characteristics}

The basic information (gender, age, occupation, job categories) of participants were obtained before the FGDs conducted from 27 participants. The majority of the respondents were $41-48$ years aged (37.04\%), male (55.56\%), and employed (77.78\%) (Table3). This means unfortunately the selected one has little information that initiates them.

Table3. Socio-demographic characteristics of middle-aged of urban residents, Ethiopia,2019

\begin{tabular}{llcr}
\hline Variable, $\mathrm{n}=27$ & Categories & Frequency $(\mathrm{n})$ & Percentage (\%) \\
\hline Age & $41-48$ years & 10 & 37.04 \\
& $49-57$ years & 9 & 33.33 \\
& $58-64$ years & 8 & 29.63 \\
\hline Gender & Male & 15 & 55.56 \\
& Female & 12 & 44.44 \\
\hline Occupation & Employed & 21 & 77.78 \\
& Unemployed & 8 & 22.22 \\
\hline Job category & Business Bankers & 2 & 7.41 \\
& Health professionals & 11 & 40.74 \\
& Teachers & 3 & 11.11 \\
& Office workers & 3 & 11.11 \\
& Self employers/less & 8 & 29.63
\end{tabular}

\subsection{Theme Categorization}

Theme 1: Barriers

The analysis applied three types of social capital: bonding (i.e. strong ties with individuals who are homogeneous in social composition, such as with family members, community sports club and close friends), bridging (i.e. weak ties with individuals who are heterogeneous in social composition, such as health care professionals) and linking such as health care system (i.e. vertical ties with the regional and federal government) (Table 4).

\section{Theme 2: Facilitators}

Fear of morbidity and mortality, limited awareness, cooperative relationships with health extension workers and motivation to participate in physical activity are identified themes. The narration of themes, categories, sub-categories materialized, and direct quotes of the participants were shown (table4).

Table4. Summary of themes, categories, direct quotes of urban residents, Ethiopia, 2019

\begin{tabular}{|l|c|l|l|}
\hline \multicolumn{2}{|c|}{ Selected codes } & Categories & Themes \\
\hline $\begin{array}{l}\text { In our home a lot of salt \& oil added, I consume } \\
\text { daily saturated oil/fat \& salt }\end{array}$ & Negative influence from family & $\begin{array}{l}\text { Bonding social } \\
\text { capital }\end{array}$ & \\
\hline Not enough vegetables \& fruits & $\begin{array}{c}\text { Inequity in demand \&supply of } \\
\text { materials }\end{array}$ & $\begin{array}{l}\text { Linking social } \\
\text { capital }\end{array}$ \\
\hline
\end{tabular}




\begin{tabular}{|c|c|c|c|}
\hline $\begin{array}{l}\text { I drink a lot to keep good relationship with my } \\
\text { friends. }\end{array}$ & Peer pressure & $\begin{array}{l}\text { Bonding social } \\
\text { capital }\end{array}$ & \multirow{9}{*}{$\begin{array}{r}\text { Barriers of } \\
\text { healthy } \\
\text { lifestyles } \\
\text { adoption }\end{array}$} \\
\hline $\begin{array}{l}\text { It is perceived as reach person for adults to get } \\
\text { obesity }\end{array}$ & $\begin{array}{l}\text { Trend /custom towards unhealthy } \\
\text { lifestyles }\end{array}$ & $\begin{array}{l}\text { Bonding social } \\
\text { capital }\end{array}$ & \\
\hline $\begin{array}{l}\text { Lack of health care workers in the community } \\
\text { health centres. }\end{array}$ & $\begin{array}{l}\text { Inequity in the allocation of public } \\
\text { resources }\end{array}$ & $\begin{array}{l}\text { Linking social } \\
\text { capital }\end{array}$ & \\
\hline $\begin{array}{l}\text { We have some know-how about healthy } \\
\text { lifestyles, but we did not have protocols }\end{array}$ & $\begin{array}{l}\text { Insufficient support from health care } \\
\text { professionals }\end{array}$ & $\begin{array}{l}\text { Bridging social } \\
\text { capital }\end{array}$ & \\
\hline Lack of community health care provider & $\begin{array}{l}\text { Inadequate training on NCD screening } \\
\text { and management }\end{array}$ & $\begin{array}{l}\text { Bridging social } \\
\text { capital }\end{array}$ & \\
\hline I didn't have a schedule for risk diagnosis & $\begin{array}{c}\text { Lack of knowledge \& skill on chronic } \\
\text { Care }\end{array}$ & $\begin{array}{l}\text { Linking social } \\
\text { capital }\end{array}$ & \\
\hline $\begin{array}{l}\text { Home nearby sports club helped me to engage } \\
\text { in regular exercise }\end{array}$ & $\begin{array}{c}\text { Motivation to participate in regular } \\
\text { exercise }\end{array}$ & $\begin{array}{l}\text { Bonding social } \\
\text { capital }\end{array}$ & \\
\hline $\begin{array}{l}\text { HEWs are good. They help us a lot with nutrition } \\
\text { education. }\end{array}$ & $\begin{array}{l}\text { Cooperative relationships with } \\
\text { health extension workers }\end{array}$ & $\begin{array}{l}\text { Bridging social } \\
\text { capital }\end{array}$ & \\
\hline $\begin{array}{l}\text { Some of us know the role of } \\
\text { adopt healthy lifestyles }\end{array}$ & $\begin{array}{l}\text { limited awareness about healthy } \\
\text { lifestyles }\end{array}$ & $\begin{array}{l}\text { Linking social } \\
\text { capital }\end{array}$ & \\
\hline
\end{tabular}

\section{Theme 1: Barrier of adopting healthy lifestyles}

Bonding social capital

1. Negative influence from family

Closely living of people influence one other in adoption of lifestyles. Both female and male participants reported that dietary decision-making to purchase and prepare for their families was the responsibility of women. Adding excess saturated/clotted oil, salt and sugar to family dish and eating an 'unhealthy diet' were common. For example, one respondent blames his assistant food cooker for inadequately added oil and salt to the dish.

"Our housemaid uses excess oil to braising onions. I quarrel with my wife. We eat visible floating fat on the dish. Always my stomach cramps and I feel heartburn after eating a meal. " (Team_3, male, 57-64 years-old group)

Female participants mentioned that substance use such as smoking, drinking alcohol, chewing chat, cannabis was shared with all family members. If someone uses substance at home, everybody in the same family develops a bad attitude.

"My husband keeps saying that it would be possible for him to fast more days but impossible to withdraw from drinking... many dependent people are living in the house, if he did not stop, we could depict in food insecure. Even with a consequence, my two boys were in line too. Our salary did not afford healthy food for the family." (Team_1, female, 41-48-year-old)

\section{Peer pressure}

People may adopt risky behaviours such as smoking, over-drinking and over-eating, as they want to be recognized by their peers. Participants believed that entertaining relatives or friends in a restaurant and drinking a lot of alcohol was necessary to earn the respect of their guests and maintain a good relationship with them.

"I have no choice...we are good friends, especially those who have been so for a long time. If they drink one bottle of alcohol, how can I just drink half?" (Team_3, female, 57-64 years-old group)

\section{Participants perceive towards Overweight and obesity}

Custom towards an unhealthy lifestyle of the members of the close network made community think that it would be all right to adopt risky behaviours. For example, most of the participants accepted that it would not be a problem for an adult to be obese. The high tolerance of family and friends towards obesity would decrease the motivation for changing behaviours. 
"We are adult enough. Being obese is a sign of doing well and it is ok for adult people. We don't care." (Team_3, male, 5764 years old)

One of the respondents elaborated, "we feel healthy. Now I am 51 years old, I have not diagnosed before. We do not have as such detail knowledge. Also cost for diagnosis is high." (Team_3, male, 57-64 years old)

"Financial reasons" emerged as a major barrier to a healthy diet. For instance, one respondent stated "not being able to afford it, money is something that can prevent you from physical activity." Some participants also stated "healthy diet cost more," "lack time to cook," and "fast food is cheaper" as barriers to healthy eating habits.(The former: Team_2, male; 49-56 years old; the latter: Team_1, female; 41-48 year-old)

\section{Accepted socialization by community}

Human beings are social creatures and they all participate in the network of social relationships. Starting from ancestors to nowadays, our community accepted coffee ceremony as a means of socialization, cultural value and welcoming guests. Preparing coffee, a minimum of two times a day and drinking at least three cups of coffee served in each period is common practice. They usually added a lot of sugar in each cup of coffee and the dose of caffeine did not measure. One person stated:

"I cannot drink coffee without added sugar, I like sweaty products like cake. Not only me, my colleagues also too. I have a cup of coffee at home only when sugar is available." (Team_1,male; 41-48 year-old)

\section{Bridging social capital}

4. Insufficient support from health care professionals Custom

Participants from three FGDs precisely explain the strong motivation of changing behaviours, although they felt that they could not succeed without professional support from health care workers. They thought that their health knowledge was too little to adopt healthy lifestyles.

"I really hope that... of course you can do an intervention here...I hope that you professionals could give us acceptable and feasible suggestions, to make us healthier. We need more knowledge to change our lifestyles. We all still like to put a lot of clotted oil and salt when cooking. We know drinking too much is harmful, but we still like drinking. We know substance use is dangerous, yet we are using few substances like chat." (Team1, female, 41-48-year-old)

\section{Inadequate awareness on NCD screening and management}

Participants illustrate poor health information coverage as a barrier to healthy lifestyles adoption: "...mass media coverage on the importance of healthy lifestyles adoption, like health and nutrition registration, radio, television (TV), newspaper, and mobile short message service (SMS) is inadequate. We think the awareness created by health care workers to the community on the health benefits of a healthy lifestyle is insufficient."(Team2, male, 49-56-year-old)

\section{Linking social capital}

1. Hospital related barriers to adopting healthy lifestyles

The respondents mentioned insufficient infrastructure and lack of professionals in the hospitals as a barrier: "...there is no aerobic and gym, active physical assessment center, metabolic syndrome therapy center, and trained professional in dieticians for nutrition council, physiotherapy and sport nutrition expert for exercise referral."(Team2, male, 49-56 year-old)

The respondents indicated the lack of physical activity protocol or guidelines prevents the use of physical activity to prevent and control NCDs:

"...physical activity tools that help health care workers by type, intensity, duration, and frequency of physical activity to be prescribed to patients is lacking in health institutes." ( Team3, male, 57-64-year-old)

2. Community-related barriers to adopting healthy lifestyles

Due to food insecurity (availability and adequacy), people practice unhealthy diet such as clotted oil. Because of this:

"I and my family consume vegetable once a week..., and occasionally fruits." (Team3, male, 57-64 years old).In addition, they consume cereal-based monotonous foods regularly. For instance, respondent raises: "we... always eat Injera with shiro wett." (Team2, male, 49-56-year-old group). Another respondent continued ...." Because of lack of time I eat unknown source food in the restaurant." (Team2, female, 49-56-year-old group)

The majority of the participants believed they did not exercise enough, while many participants acknowledged the importance of regular physical activity. Most of the respondents gave simple and concise responses such as 
"yes [don't have enough exercises]" and "I feel like I do not have enough activities." Some participants elaborated that their beliefs were the reasons for physical inactivity. For example, one participant said "I don't have enough exercise. I feel like I don't have sufficient time for exercise, I work." (Team1, male,41-48 years old)

"Financial reasons" emerged as a major barrier to a healthy diet. For instance, one person stated, "not being able to afford it, money is something that can prevent you from physical activity." Some participants also stated "healthy food cost more," "[being] busy," and "fast food, literally fried biscuit is cheaper" as barriers to healthy eating habits. Lack of free time was revealed as a key subtheme relating to insufficient enough regular exercise. "Time," "work" and "lack of awareness" were common responses when asked about what prevented participants from exercising.

3. Inequity in the allocation of public resources

Inequity in the allocation of public resources: due to lack of incorporating healthy lifestyle adoption into urban health policy to access good quality of health services. Participants complained that the city did not have community health care workers other than HEWs.

"Generally speaking, (unlike developed countries), our doctors are not so impolite, but the problem is too few doctors and busy by routine work. There is less attention to NCD prevention through community-based healthy lifestyles education." (Team_2, male, under 49-56 years-old)

4. Barrier related to healthcare professionals to adopt healthy lifestyles

One respondent indicated that the lifestyles of healthcare providers is the other barrier to adopt it into their work: "...most healthcare providers may not have active lifestyles may be due to lack of time, skill, confidence, knowledge, and prioritizing clinical matters which may enable them not to use physical exercise in the healthcare system."(Team1, male, under 41-48years-old)

The respondents reflect the experience of health care workers associated with healthy lifestyles adoption is not a model for the community. A male participant indicated;

"...experience can be a barrier to integrating physical exercise in the healthcare to treat NCDs. For instance, lack of practical experience on the type, duration, intensity, and frequency of exercise to be integrated with healthcare to prevent and control NCDs is barriers among healthcare professionals." (Team3, male, under 57-64years-old)

\section{Theme_2: Opportunities to adopt healthy lifestyles}

\section{Bonding social capital}

\section{Motivation to participate in regular exercise}

Motivation to engage in regular exercise occurred when participants had many friends who have similar interest. Sports clubs, such as riding a horse, running, swimming, usually involved in many people with similar health goals. Participants remarked that the community team sports helped them to engage in physical activity.

"Yes! When I was young, I had to look, but my home is far from a sports club, so it was impossible to participate in a community sports club. Due to opportunity living in condominium (home nearby sport club), it helped me engage in regular exercise."(Team3, female, 57-64-year-old)

\section{Bridging social capital}

2. Cooperative relationships with health extension workers

Respondents mentioned the cooperative relationship with health extension workers, encouraging their health-seeking behaviour and enhancing healthy lifestyles campaigns. "Health extension workers cooperate with each other in our town." (Team1, male, 41-48 years old) "The health extension workers are very nice! Just like our family. We like them." (Team2, female, 49-56year old)

Linking social capital

\section{Fear of morbidity, mortality and medical help-seeking}

An important factor that appears from the participants was fear of morbidity and mortality due to symptomatic degenerative diseases and participants take care of themselves. They had an understanding that by keeping the levels under control, it could be prevented. All the participants agree on the importance of early screening before become ill. Nevertheless, due to the expensive, limited knowledge and time-consuming hospital visits, they could not afford frequent follow-ups. Hence, most of them did not visit a doctor unless they were very sick. One person expressed the fear and his experiences as follow:

"I take care of my lifestyles modification, medications and checking my sugar levels before every meal... I don't want to die early. I want to live for my family." (Team1, male, 41-48 years old) 


\section{Limited awareness about healthy lifestyles and NCDs}

Linking social capital included awareness of healthy lifestyles nationally. Participants stated the levels of awareness were limited due to various health policies and its implementation.

"Formerly, I didn't know it (healthy lifestyles), now I understand how important it is. Nowadays, we have limited knowledge of it; some of us hear it through TV and radio, and health extension workers. We all know what should be done in order to live longer, but in the old days, nobody heard of that." (Team3, female, 57-64year old).

\section{Discussion}

We identified barriers and facilitators in adopting a healthy lifestyle among urban residents in west Ethiopia. Bonding, bridging and linking social capital would work as facilitators to adopt healthy lifestyles, as seen in awareness creation and made cooperative relationship among health extension workers. Conversely, social capital also works as a barrier, such as perceived barriers to adopt a healthy lifestyle like "financial reasons" emerged as a major barrier to healthy diet and peer pressures to continue over-drinking. This encourages the adoption of a healthy lifestyle at the family as well as community level.

This study reveals the following findings. First, the lack of affordable unsaturated oil, lack of fruit and vegetable availability and economic problems to purchase were major barriers to adopt a healthy diet. Second, lack of knowledge and skill on chronic Care (For instance: barrier related to participants believed getting obese at an adult age is normal). Finally, the lack of community health care workers and inequity in the allocation of public resources were observed. Our study narrated barriers and opportunities in adopting healthy lifestyles as of domains stated in focus group guides.

Barriers related to diet was raise from lack of knowledge, economy and availability of resources like unsaturated oil, fruits and vegetables. Due to food insecurity, most people consume saturated oil and cereal-based monotonous food. Also, almost all respondents indicated they did not eat fruits because of their expensiveness and unavailability. For example, $1 \mathrm{Kg}$ of apple and banana were 100 and 30 Ethiopian birr, respectively. This result was similar to the finding of perception and practice of 'healthy diet in relation to non-communicable diseases (Nagoya, et al. 2018).

As urbanization increases physical activity decreases and sedentary life increases. In healthcare, lacks practical guidelines on how to prescribe physical exercise other barriers. Previous research demonstrated that healthcare professionals who use physical exercise guidelines, use physical exercise to treat NCDs patients more confidently than those who are not using it (Brien, et al. 2017).

The findings confirm that there are barriers regarding marginalizing professionals from hospitals, lack of chronic health workers, and physical activity prescription hindering risk factors. Similar study conducted supporting the idea with lack of physical activity protocol in the Ethiopian health care system possibly imply the lack of knowledge about the health benefits of physical activity among policymakers and absence of physical activity as a strategy for NCDs prevention in the national health policy (Melkamu et al. 2018). In contrasting a study in America reported the presence of guidelines and physical exercise in all medical schools of the USA (Lobelo, et al. 2014).

Bonding social capital provides intensive supports to the individuals (Szreter \& Woolcock M, 2004). It is expected to play a significant role in adopting healthy lifestyles, such as reducing salt intakes and exercising regularly. Several studies indicated the role of social capital in adherence to healthy lifestyles. For example, a Dutch study shows that people with a high level of neighbourhood social capital were more likely to be physically active and non-smoker (Mohnen et al., 2012). As of (WHO 2010) on physical activity: $23 \%$ of adults aged greater than 18 years insufficiently engage in moderate-intensity physical activity (< 150 minutes) per week.

In developed countries, NCD services are preferably delivered in an established NCD clinic with a team of experts. However, our current placement of health facility structure and staffing is impossible to have liked this ideal scenario. It is recommended to integrate healthy lifestyles education into health post with primary prevention of diseases.

We identified barriers with respect to the health system, patients and professional to prevent and control NCDs. Health care workers/ general practitioners did not prescribe healthy diet and physical activity because of the absence of protocol. The community have limited knowledge to become diagnosis early identification of risks factors of chronic diseases. The study conducted in Sydney focused on particular lifestyle risk factors of obesity, tobacco and alcohol support our study. Due to Lack of dedicated clinical guidelines and protocols for primary care, only a small proportion of primary care visits include risk assessment, provision of information or support for behavioural change (Harris, 2009).

To sum up, lack of promoting healthy lifestyles through media alone, improper placement of health facility structure and staffing, lack of community coaching, unavailability of (fruits \& vegetable, unsaturated oil, salt adequacy measurement), food price inflation, and lack of public awareness creation do not secure without coaching leadership implementation and policy advocacy.

Page | 26 


\section{Conclusion}

Our findings demonstrated that bonding, bridging and linking social capital could work as barriers and facilitators for adopting healthy lifestyles in urban residents of western Ethiopia. Due to different barriers stated in the above social capital frameworks, the community's unhealthy lifestyles are practised. So, it implies face to face education needed to adopt healthy lifestyles and prevent non-communicable diseases.

\section{Implications}

The results of this study bring valuable insights for the further development of healthy lifestyles education. Financial reasons and the lack of available time were the main determinants for people to engage in a healthy diet and physical activity. The government needs to undertake food insecurity. Increasing the availability of affordable healthy foods like polyunsaturated fatty acid oil, fruits \& vegetables, and opportunities for physical activities in communities is essential for healthy lifestyles. Another bottleneck is the lack of community health care workers that give awareness on the effect of healthy lifestyles education on metabolic syndrome and quality of life. Due to the lack of health and nutrition information desks in all institutes, people also have limited knowledge on early screening, prevention and self-management of NCDs.

The identified barriers are indicators for policymakers to plan, design, and implement healthy lifestyles adoption into the chronic care setting and redesign the existing health policy. Besides that, training of health care workers on healthy lifestyles adoption, physical exercise prescription and metabolic syndrome prevention at all levels of medical education is required. Additionally, establishing communal training and a therapy centre will be needed.

Multimodal behavioural intervention, including education, medical nutrition therapy (diet, social, physio and psycho) and coping strategies, need attention to NCD prevention. The nutrition lens approach is required to remove the barriers and use opportunities for healthy lifestyles adoption. Whatever active screening is recommended for all individuals above 40years, this scenario needs paradise shift to be mid of life expectance (above 30 years and above 20 years for those of their family who have a history of NCDs). Our people do not visit health institutes to detect NCD risks early; so, community mobilization and motivation are suggested. Make sure noticing people habitual practices may have health implication to bring behavioural changes.

Ethiopia current health care system placement of health facility structures and staffing is not established NCD clinic with a team of experts, except the specialized hospitals. So, it is strongly recommended to integrate healthy lifestyles education to the health post for primary prevention of NCD through health extension workers under the supervision of health officer or practitioner nurses, deliver NCD services at ART clinic, chronic care/referral clinic and specialized clinic in the health centre, general hospital and tertiary hospital respectively. To be effective in healthy lifestyles adoption and chronic non communicable diseases care, Ethiopia should take social capital perspective into account and implement a primary prevention plan using health care worker.

Acknowledgements: We would like to thank Jimma University for granting ethical approval and funding the study. Furthermore, our heart fell thank go to all study participants for their commitment and spent their valuable time providing the required information to complete this study. Moreover, we thank Jimma University for funding the study.

Conflict of interest: There is no competing interest associated with the publication of this manuscript. References

[1] Abegunde DO, Mathers CD, Adam T, Ortegon M, Strong K. The burden and costs of chronic diseases in low-income and middle-income countries. Lancet.2007;370(9603):1929-38.

[2] Asnakew Achaw Ayele1*, Yohannes Kelifa Emiru2, Sofonyas Abebaw Tiruneh3, Belete Achamyelew Ayele4, Alemayehu Digssie Gebremariam3 and Henok Getachew Tegegn1,5(2018). Level of adherence to dietary recommendations and barriers among type 2 diabetic patients: a cross-sectional study in an Ethiopian hospital. Ayele et al. Clinical Diabetes and Endocrinology (2018) 4:21. https://doi.org/10.1186/s40842-018-0070-7.

[3] Brien, M. W. O., Shields, C. A., Oh, P. I., \& Fowles, J. R. (2017). Health care provider confidence and exercise prescription practices of Exercise is Medicine Canada workshop attendees. NRC Research Press, 42(14 December), 384-390.

[4] Danaei G, Finucane MM, Lin JK, Singh GM, Paciorek CJ, Cowan MJ, et al. National, regional, and global trends in systolic blood pressure since 1980: systematic analysis of health examination surveys and epidemiological studies with 786 country-years and 5.4 million participants. Lancet. 2011;377(9765):568-77.

[5] Degu G, Tegbar Y. (2006), Research methodology lecture note For Health Science Students, Gondar University.

[6] Dietz, W.H., R.C. Brownson, C.E. Douglas, J.J. Dreyzehner, R.Z. Goetzel, S.L. Gortmaker, J.S. Marks, K.A. Merrigan, R.R. Pate, L.M. Powell, and M. Story. 2016. Improving Physical Activity and Nutrition and Reducing Tobacco Use and Obesity to Prevent Chronic Disease. Discussion Paper, Vital Directions for Health and Health Care Series. National Academy of Medicine, Washington, DC. https://nam.edu/wpcontent/uploads/2016/09/chronic-disease-prevention-tobacco-physical-activity-and-nutrition-for-a-healthy-start.pdf.

[7] Edris abda1 leja hamza2 Fasil Tessema3 Waqtola cheneke4,2016. Metabolic syndrome and associated factors among outpatients of Jimma University Teaching hospital, Diabetes, Metabolic Syndrome and Obesity: Targets and Therapy 2016:9. https://www.dovepress.com/ by 213.55.92.81 on 22-Oct-2017. 
[8] Engelen, L., Bauman, A., Bellew, B., Caillaud, C., Merom, D., Singh, M. F., ... Network, A. (2017). Capacity building in physical activity and noncommunicable disease prevention: a low-cost online training course can reach isolated practitioners. Global Health Promotion, 24(1), 27-33. https://doi.org/10.1177/1757975915586957.

[9] Ethiopia Demographic and Health Survey (EDHS,2011). Central Statistical Agency. Addis Ababa, Ethiopia and ICF International Calverton, Maryland, USA.

[10] Fatt, Q. K. (2016). Non-Communicable Diseases: A Major Problem Worldwide. PublicHealth - Open JournalOpen Journal, 1(2), 32-35. https://doi.org/10.17140/PHOJ-1-107.

[11] Girum Tefera, 2015. Determinants of Proteinuria among Type 2 Diabetic Patients at Shakiso Health Center, Southern Ethiopia: A Retrospective Study. Advances in Diabetes and Metabolism 2(3): 48-54, 2014 http://www.hrpub.org. DOI:10.13189/adm.2014.020302.

[12] Global strategy on diet, physical activity and health. Geneva: WHO; 2004 (http://www.who.int/dietphysicalactivity/strategy/eb11344/strategy_english_web.pdf, accessed 13 June 2016).

[13] Grundy SM, Cleeman JI, Daniels SR, et al. Diagnosis and management of the metabolic syndrome: an American Heart Association/National Heart, Lung, and Blood Institute Scientific Statement. Circulation 2005;112:2735-52.

[14] Harris M. The role of primary health care in preventing the onset of chronic disease, with a particular focus on the lifestyle risk factors of obesity, tobacco and alcohol. Sydney: Centre for Primary Health Care and Equity, University of New South Wales; 2009 (http://www.preventativehealth.org.au/internet/preventativehealth/publishing.nsf/ Content/0FBE203C1C547A82CA257529000231BF/\$File/commpaper-primary-hlth-care-harris.pdf, accessed 2 June 2016).

[15] Herman, W.H., Hoerger, T.J., Brandle, M., Hicks, K., Sorenson, S., Zhang, P., et al. The costeffectiveness of lifestyle modification or metformin in preventing type 2 diabetes in adults with impaired glucose tolerance. Ann Intern Med. 142, 323-332, 2005.

[16] Lemlem Weldegerima Gebremariam1, Atsuko Aoyama1, Alemayehu Bayray Kahsay2, Yoshihisa Hirakawa1, Chifa Chiang1, Hiroshi Yatsuya1, 3, and Akiko Matsuyama(2018). Perception and practice of 'healthy' diet in relation to noncommunicable diseases among the urban and rural people in northern Ethiopia: a community-based qualitative study. Nagoya J. Med. Sci. 80. 451-464, 2018. doi:10.18999/nagjms.80.4.451.

[17] Lobelo, F., Stoutenberg, M., \& Hutber, A. (2014). The Exercise is Medicine Global Health Initiative: A 2014 update. British Journal of Sports Medicine, 48(22), 1627-1633.https://doi.org/10.1136/bjsports-2013-093080.

[18] Melkamu D. Kassa1 \& Jeanne Grace1(2018). Barriers to Integrate Physical Exercise Into the Ethiopian Healthcare System to Treat NonCommunicable Diseases. Global Journal of Health Science; Vol. 10, No. 10; 2018 ISSN 1916-9736 E-ISSN 1916-9744 .Published by Canadian Center of Science and Education.

[19] Mensah, G. A. (2016). Tackling Noncommunicable Diseases in Africa. Health Education \& Behavior, 43(1s), 7S-13S https://doi.org/10.1177/1090198116633454.

[20] Mohnen SM, Volker B, Flap H, Groenewegen PP. Health-related behavior as a mechanism behind the relationship between neighborhood social capital and individual health - a multilevel analysis. Bmc Public Health, 2012; 12.

[21] Namey E, Guest G, Thairu L et al. Data reduction techniques for large qualitative data sets. In: Guest G, MacQueen KM (eds). Handbook for Team-Based Qualitative Research. Lanham, MD: AltaMira Press, 2007, 137-162.

[22] Sajwani RA, Shoukat S, Raza R, Shiekh MM, Rashid Q, Siddique MS, et al. Knowledge and practice of healthy lifestyle and dietary habits in medical and non-medical students of Karachi, Pakistan. J Pak Med Assoc. 2009;59:650-5.

[23] Sallis, R. (2017). Exercise is medicine: a call to action for physicians to assess and prescribe exercise exercise. ThePhysician and Sportsmedicine, 43(1), 22-26. https://doi.org/10.1080/00913847.2015.1001938.

[24] Spark, Arlene J. Nutrition in public health : principles, policies, and practice / author, Arlene Spark. p. ; cm. ISBN-13: 978 0-8493-1473-5 (hardcover : alk. paper) ISBN-10: 0-8493-1473-9 (hardcover : alk. paper). Hunter University, NewYork ,U.S.A. 2007 by Taylor \& Francis Group, LLC.

[25] Szreter S, Woolcock M. Health by association? Social capital, social theory, and the political economy of public health. International Journal of Epidemiology, 2004; 33: 650-667.

[26] Taylor, D. (2014). Physical activity is medicine for older adults. Postgraduate Medical Journal, 90(1059), 26-32. https://doi.org/10.1136/postgradmedj-2012-131366.

[27] WHO. Global Status Report on Noncommunicable Diseases. Geneva: World Health Organization; 2010.

[28] Worku A, Abebe SM, Wassie MM. Dietary practice and associated factors among type 2 diabetic patients: a cross sectional hospital based study, Addis Ababa, SpringerPlus 2015;4:15.

[29] World Health Organizatio [WHO]. (2015). Noncommunicable Diseases Progress Monitor. https://doi.org/10.2766/120051.

[30] Zhang Y, Ma D, Cui R, Hilawe EH, Chiang C, Hirakawa Y, et al. Facilitators and barriers of adopting healthy lifestyle in rural China: a qualitative analysis through social capital perspectives. Nagoya J Med Sci, 2016; 78: 163-173. 